\title{
General relativistic nonideal fluid equations for dark matter from a truncated cumulant expansion
}

\author{
Alaric Erschfeld, ${ }^{*}$ Stefan Floerchinger, ${ }^{\dagger}$ and Maximilian Rupprecht ${ }^{\ddagger}$ \\ Institut für Theoretische Physik, Ruprecht-Karls-Universität Heidelberg Philosophenweg 16, \\ D-69120 Heidelberg, Germany
}

(Received 6 June 2020; accepted 21 August 2020; published 16 September 2020)

\begin{abstract}
A new truncation scheme based on the cumulant expansion of the one-particle phase-space distribution function for dark matter particles is developed. Extending the method of moments in relativistic kinetic theory, we derive evolution equations which supplement the covariant conservation of the energymomentum tensor and particle number current. Truncating the cumulant expansion we obtain a closed, covariant and hyperbolic system of equations which can be used to model the evolution of a general relativistic nonideal fluid. As a working example we consider a Friedmann-Lemaitre-RobertsonWalker cosmology with dynamic pressure and solve for the time evolution of the effective equation of state parameter.
\end{abstract}

DOI: 10.1103/PhysRevD.102.063520

\section{INTRODUCTION}

Observations indicate that a substantial part of the energy budget of the Universe is made up of dark matter. To a good approximation, it seems to be noninteracting except through gravitational interactions [1,2]. While there are various ideas as to the nature of dark matter, there is no verified microscopic theory so far. In the absence of a direct detection, its properties can only be constrained through cosmological and astrophysical observations. These are necessarily observations in the macroscopic domain, while one would ultimately be interested in a microscopic understanding in terms of a quantum field theory.

In order to learn more about the properties of dark matter, one therefore turns to effective descriptions, such as fluid approximations or variants of kinetic theory. While isotropy and homogeneity constrain the cosmological background dynamics to be of perfect fluid type [3], gravitational collapse is caused by fluctuations which are expected to generate nonvanishing shear stress at smaller scales and therefore require a nonideal fluid description. For a consistent coupling to gravity through the Einstein field equations and in order to have a large scale description that obeys relativistic causality, one should aim for a

\footnotetext{
*erschfeld@thphys.uni-heidelberg.de

floerchinger@thphys.uni-heidelberg.de

"rupprecht@thphys.uni-heidelberg.de
}

Published by the American Physical Society under the terms of the Creative Commons Attribution 4.0 International license. Further distribution of this work must maintain attribution to the author(s) and the published article's title, journal citation, and DOI. Funded by SCOAP ${ }^{3}$. relativistic fluid description. This is also important to understand the possible interplay with modified theories of gravity.

Another striking example for the applicability of relativistic fluid dynamics are heavy-ion collisions, such as those studied at the Relativistic Heavy Ion Collider (RHIC) and the Large Hadron Collider (LHC). At macroscopic scales these exhibit collective fluidlike behavior, such that the bulk dynamics can be described in terms of relativistic nonideal fluid dynamics. This should be understood as a low-energy effective description of quantum chromodynamics in an out-of-equilibrium situation of high energy density [4-7].

First attempts to construct a theory of relativistic fluid dynamics were made by Eckart [8] and later by Landau and Lifschitz [9]. However, these are known to be unstable and acausal [10-12] and can even have modes which propagate faster than light [13]. In general, many so-called first-order theories suffer from the fact that the equations of motion are parabolic in structure making them acausal [14]. Only recently, a class of first-order theories has been proposed that does not suffer from these problems [15-18]. While the energy-momentum tensor is expanded to first order in gradients around the ideal fluid form, this is done in such a way that the resulting equations of motion are second-order hyperbolic differential equations and can therefore obey relativistic causality.

As another possibility to remedy these issues, extended theories have been proposed. In general, hydrodynamical descriptions are possible due to conservation laws. In the relativistic context, this is the covariant conservation of the energy-momentum tensor, sometimes supplemented by a covariantly conserved particle number current. In such a 
framework one has 10 degrees of freedom in the components of the energy-momentum tensor and four in the particle number current, but only five equations of motion from energy-momentum and particle number conservation. Assuming that all the degrees of freedom are independent and dynamical, the system of equations is not closed and additional information is needed.

The standard theory of hydrodynamics [9] provides such additional relations from an expansion of the conserved currents in terms of gradients of the fields that govern an ideal fluid, namely the fluid velocity and thermodynamic variables such an temperature and chemical potential. At a given finite order, this leads to additional constraint equations that related the components of the conserved currents to the hydrodynamic fields. Such a gradient expansion is particularly well motivated in the vicinity of thermal equilibrium or when interaction effects are so strong that they quickly drive the system back towards local equilibrium when the latter is violated as a consequence of fluid motion.

In the context of dark matter, this close-to-equilibrium gradient expansion is not particularly well motivated, especially at late times and at small scales. Indeed, when interactions besides gravity are absent, one cannot assume that local thermal equilibrium is restored quickly. However, additional information to close the system of equations can also be provided in the form of additional evolution equations. This leads to a formalism with more dynamical variables or fluid fields, for example shear stress, bulk viscous pressure, heat current or particle diffusion current.

Historically, such additional evolution equations have been first obtained from a kinetic theory approach where the system is characterized by a phase-space distribution function which obeys the relativistic Boltzmann equation. With the method of moments [19] one can derive additional equations of motion in order to evolve the dynamical degrees of freedom and close the system by a systematic truncation, such as a derivative expansion. This has been done, most notably in the nonrelativistic case by Müller [20] and in the relativistic case by Israel and Stewart $[21,22]$, keeping terms up to second order in gradients, socalled second-order theories. More modern approaches include derivative expansions, including all terms of second order in gradients [23] and formulations derived from the relativistic Boltzmann equation, keeping all terms in the moment expansion [24]. Recent applications of such approaches include Refs. [25-31].

In the cosmological context, relativistic fluid dynamics have been extensively studied. These investigations range from ideal fluid dynamics [32,33], to second-order theories [34-36] and to the Einstein-Vlasov system of equations [37-39]. Further there have been investigations of effective fluid theories [40-42] and more recently a statistical field theory which maps to a nonequilibrium kinetic theory has been developed [43-45].
In the work presented here, we essentially adapt the method of moments and study a truncation of the cumulant expansion of the phase-space distribution function for dark matter particles without collisions. In particular, we concentrate on a description of dark matter as classical particles. A more direct relation of the fluid picture to a quantum field theoretic description is left for future work (see also Refs. [46,47]).

In Sec. II we present the relativistic Vlasov equation, which governs the evolution of the one-particle phasespace distribution function. We define moments and cumulants and study their behavior under the Vlasov equation. Here, the first and second moment are the particle number current and energy-momentum tensor, respectively. We propose a truncation scheme in terms of the cumulant expansion of the distribution function and explicitly perform this truncation after the first and second cumulant. For these one can reconstruct a modified version of the phasespace distribution function. We discuss the nonconsistency of these truncations, in the sense that they are not preserved by the Vlasov equation.

In Sec. III we derive closed equations of motion for the dynamical fluid fields parametrizing the 14 independent degrees of freedom. To this end we use the covariant conservation of the first three moments and close the system of equations by neglecting the third cumulant. We discuss the hyperbolic structure of the system of equations, allowing for a causal description provided the characteristic propagation speeds are finite. By this we construct a closed, covariant and hyperbolic system of partial differential equations which can be used to model a general relativistic nonideal fluid. Finally, we present a simple application of this truncation in the form of a Friedmann-LemaîtreRobertson-Walker (FLRW) cosmology.

Throughout this paper, except when otherwise stated, we work in natural units where $c=\hbar=k_{\mathrm{B}}=1$.

\section{RELATIVISTIC KINETIC THEORY}

\section{A. The relativistic Vlasov equation}

The kinetic theory for classical point particles can be formulated in general coordinates $x$ and in curved spacetime. ${ }^{1}$ In the absence of any force except for gravity, particles follow geodesics,

$$
\frac{\mathrm{d}^{2} x^{\mu}}{\mathrm{d} s^{2}}+\Gamma_{\rho \sigma}^{\mu} \frac{\mathrm{d} x^{\rho}}{\mathrm{d} s} \frac{\mathrm{d} x^{\sigma}}{\mathrm{d} s}=0,
$$

where $s$ is an affine parameter and $\Gamma_{\rho \sigma}^{\mu}(x)$ are the Christoffel symbols of the second kind of the space-time metric tensor $g_{\mu \nu}(x) .{ }^{2}$ We denote the components of four-vectors by

\footnotetext{
${ }^{1}$ For simplicity we assume the absence of the corresponding antiparticles.

${ }^{2}$ We work with the metric signature $(-,+,+,+)$.
} 
Greek letters and summation over the same covariant and contravariant indices is understood. The state of the theory is described by a one-particle phase-space distribution function $f(x, p)$. Here, the four-momentum for particles of mass $m$ is $p^{\mu}=m \mathrm{~d} x^{\mu} / \mathrm{d} s$.

The number of particles at position $x$, in the momentum range $\sqrt{g} \mathrm{~d}^{4} p /(2 \pi)^{4}$ and in a mass range $\mathrm{d} m$ flowing through the hypersurface element

$$
\mathrm{d} \Sigma_{\mu}(x)=\frac{\sqrt{g(x)}}{3 !} \epsilon_{\mu \nu \rho \sigma} \mathrm{d} x^{\nu} \mathrm{d} x^{\rho} \mathrm{d} x^{\sigma},
$$

is given by

$$
\begin{aligned}
\mathrm{d} N= & \xi(m) \mathrm{d} m p^{\mu} \mathrm{d} \Sigma_{\mu}(x) \sqrt{g(x)} \frac{\mathrm{d}^{4} p}{(2 \pi)^{4}} \\
& \times 4 \pi \theta\left(p^{0}\right) \delta\left(p^{2}+m^{2}\right) f(x, p) .
\end{aligned}
$$

Here $g(x)=-\operatorname{det}\left(g_{\mu \nu}(x)\right)$ is the determinant of the metric tensor, $\epsilon_{\mu \nu \rho \sigma}$ is the total antisymmetric Levi-Civita symbol and we abbreviate the space-time inner product as $p^{2}=$ $g_{\mu \nu} p^{\mu} p^{\nu}$. Further $\delta$ and $\theta$ denote the (one-dimensional) Dirac delta and Heaviside unit step function, respectively. For fixed mass $m$, the momenta fulfill the on-shell constraint $p^{2}+m^{2}=0$, but we allow more generally a distribution of masses $\xi(m)$ which we take to be independent of time and space. As an example for a dark matter model with some distribution of masses one may think about primordial black holes. For a single species of particles with unique mass $m_{*}$ one has $\xi(m)=\delta\left(m-m_{*}\right)$.

The particle number current is given by

$$
N^{\mu}(x)=\int_{m} \xi(m) \int_{p} p^{\mu} 4 \pi \theta\left(p^{0}\right) \delta\left(p^{2}+m^{2}\right) f(x, p),
$$

and the energy-momentum tensor is

$$
T^{\mu \nu}(x)=\int_{m} \xi(m) \int_{p} p^{\mu} p^{\nu} 4 \pi \theta\left(p^{0}\right) \delta\left(p^{2}+m^{2}\right) f(x, p) .
$$

Here and throughout we abbreviate the mass and momentum integrals as

$$
\int_{m}=\int_{0}^{\infty} \mathrm{d} m, \quad \int_{p}=\sqrt{g} \int_{\mathbb{R}^{4}} \frac{\mathrm{d}^{4} p}{(2 \pi)^{4}} .
$$

In the absence of scattering, the distribution function is conserved along a geodesic, $\mathrm{d} f / \mathrm{d} s=0$, which implies the Vlasov equation [48,49],

$$
\left[p^{\mu} \frac{\partial}{\partial x^{\mu}}-\Gamma_{\rho \sigma}^{\mu} p^{\rho} p^{\sigma} \frac{\partial}{\partial p^{\mu}}\right] f=0
$$

Note however that this equation is somewhat more general than usual because $f(x, p)$ is here not restricted to $p^{2}+m^{2}=0$ being satisfied for a unique value of $m$. We allow a more general distribution of masses such that also the distribution function $f(x, p)$ is more general, but Eq. (7) still determines its time evolution.

In the following it is convenient to introduce the modified distribution function,

$$
\tilde{f}(x, p)=\int_{m} \xi(m) 4 \pi \theta\left(p^{0}\right) \delta\left(p^{2}+m^{2}\right) f(x, p),
$$

such that for example $T^{\mu \nu}=\int_{p} p^{\mu} p^{\nu} \tilde{f}(x, p)$. Equation (7) immediately implies that the modified distribution function also obeys the Vlasov equation,

$$
\left[p^{\mu} \frac{\partial}{\partial x^{\mu}}-\Gamma_{\rho \sigma}^{\mu} p^{\rho} p^{\sigma} \frac{\partial}{\partial p^{\mu}}\right] \tilde{f}=0 .
$$

\section{B. The method of moments and cumulants}

Often one is not interested in the full distribution function $f(x, p)$ but rather in its moments and cumulants. In analogy to probability distributions one can define fully symmetric four-momentum moments,

$$
\begin{aligned}
M^{\mu_{1} \cdots \mu_{n}}(x) & =\int_{m} \xi(m) \int_{p} p^{\mu_{1}} \cdots p^{\mu_{n}} 4 \pi \theta\left(p^{0}\right) \delta\left(p^{2}+m^{2}\right) f(x, p) \\
& =\int_{p} p^{\mu_{1}} \cdots p^{\mu_{n}} \tilde{f}(x, p)
\end{aligned}
$$

the set of which completely characterize the distribution function. These can be conveniently derived from a moment-generating function,

$$
\begin{aligned}
z(x ; l) & =\int_{m} \xi(m) \int_{p} \mathrm{e}^{l_{\mu} p^{\mu}} 4 \pi \theta\left(p^{0}\right) \delta\left(p^{2}+m^{2}\right) f(x, p) \\
& =\int_{p} \mathrm{e}^{l_{\mu} p^{\mu}} \tilde{f}(x, p),
\end{aligned}
$$

by $n$-fold differentiation with respect to the source fourvector $l_{\mu}$. The normalization $z(x)=z(x ; 0)$ is the zeroth moment and it is evident from Eqs. (4) and (5) that the particle number current $N^{\mu}(x)$ and the energy-momentum tensor $T^{\mu \nu}(x)$ are the first and second moment, respectively. Due to the on-shell Dirac delta function in Eq. (10) one obtains the relation

$$
g_{\rho \sigma} M^{\rho \sigma \mu_{1} \cdots \mu_{n}}+\int_{m} \xi(m) m^{2} \int_{p} p^{\mu_{1}} \cdots p^{\mu_{n}} 4 \pi \theta\left(p^{0}\right) \delta\left(p^{2}+m^{2}\right) f(x, p)=0,
$$


which in the limit of a single particle species with unique mass $m$ reads

$$
g_{\rho \sigma} M^{\rho \sigma \mu_{1} \cdots \mu_{n}}+m^{2} M^{\mu_{1} \cdots \mu_{n}}=0,
$$

and relates moments which differ by two orders. For a spectrum of masses it is in general not possible to express the relation (12) uniquely in terms of finite moments.

Similarly the distribution function is completely characterized by the connected parts of the moments, the socalled cumulants $C^{\mu_{1} \cdots \mu_{n}}(x)$. These can be derived from the cumulant-generating function $\ln (z(x ; l))$ in the same manner as moments are derived from $z(x ; l)$.

The first few cumulants and moments are related by the expressions

$$
\begin{aligned}
N^{\mu} & =z C^{\mu}, \\
T^{\mu \nu} & =z\left(C^{\mu \nu}+C^{\mu} C^{\nu}\right), \\
M^{\mu \nu \rho} & =z\left(C^{\mu \nu \rho}+3 C^{(\mu \nu} C^{\rho)}+C^{\mu} C^{\nu} C^{\rho}\right),
\end{aligned}
$$

and vice versa

$$
\begin{aligned}
C^{\mu} & =\frac{1}{z} N^{\mu}, \\
C^{\mu \nu} & =\frac{1}{z} T^{\mu \nu}-\frac{1}{z^{2}} N^{\mu} N^{\nu}, \\
C^{\mu \nu \rho} & =\frac{1}{z} M^{\mu \nu \rho}-\frac{3}{z^{2}} T^{(\mu \nu} N^{\rho)}+\frac{2}{z^{3}} N^{\mu} N^{\nu} N^{\rho} .
\end{aligned}
$$

Here we denote the symmetrization with respect to a set of indices by a pair of parentheses around them. Similar relations hold for higher order moments and cumulants and can be straightforwardly derived from the corresponding generating functions. Combining the relations (14) and (15) one can express the $n$th moment in terms of the lower order moments and the $n$th cumulant,

$$
\begin{aligned}
N^{\mu} & =z C^{\mu}, \\
T^{\mu \nu} & =\frac{1}{z} N^{\mu} N^{\nu}+z C^{\mu \nu}, \\
M^{\mu \nu \rho} & =\frac{3}{z} T^{(\mu \nu} N^{\rho)}-\frac{2}{z^{2}} N^{\mu} N^{\nu} N^{\rho}+z C^{\mu \nu \rho} .
\end{aligned}
$$

The Vlasov equation (7) implies the covariant conservation of all moments,

$$
\nabla_{\nu} M^{\nu \mu_{1} \cdots \mu_{n}}=0,
$$

where $\nabla_{\mu}$ denotes the covariant derivative with respect to the coordinates $x^{\mu}$. It is immediately clear that the evolution equations are independent of each other at each order. ${ }^{3}$ In contrast, the cumulants follow the nonlinear evolution equation,

\footnotetext{
${ }^{3}$ However, note that the on-shell constrains (12) yield additional relations.
}

$$
\nabla_{\nu} C^{\nu \mu_{1} \cdots \mu_{n}}+\sum_{S} C^{\alpha_{1} \cdots \alpha_{|S|} \nu} \nabla_{\nu} C^{\alpha_{|S|+1} \cdots \alpha_{n}}=0
$$

where the sum runs over all combinations of picking indices $\left\{\alpha_{1}, \ldots, \alpha_{n}\right\}$ out of $\left\{\mu_{1}, \ldots, \mu_{n}\right\}{ }^{4}$ The nonlinear terms in the evolution equation of the $n$th cumulant involve all lower order cumulants and the sum of orders of the two cumulants in the quadratic terms always adds to $n$. To discuss truncations of the cumulant expansion, it is useful to rewrite Eq. (18) into a more appropriate form,

$C^{\nu} \nabla_{\nu} C^{\mu_{1} \cdots \mu_{n}}+\nabla_{\nu} C^{\nu \mu_{1} \cdots \mu_{n}}+\sum_{S}^{\prime} C^{\alpha_{1} \cdots \alpha_{|S|} \nu} \nabla_{\nu} C^{\alpha_{|S|+1} \cdots \alpha_{n}}=0$,

where the prime indicates that the sum now excludes the case of distributing all indices to the second cumulant. ${ }^{5}$ Here, the part parallel to the fluid four-velocity of the derivative operator $C^{\nu} \nabla_{\nu}$ can be understood as the relativistic analog of the convective derivative, such that Eq. (19) serves as an equation of motion for the $n$th cumulant. We remark that Eq. (19) involves all cumulants of lower and the next higher order. This structure sets strong restrictions for a consistent truncation of the cumulant expansion as we discuss at the end of the next section.

In the nonrelativistic limit this infinite tower of equations creates the so-called Vlasov hierarchy [50-52]. This can be made explicit by taking the nonrelativistic limit of the moments (10), which for simplicity we do for a single particle species of unique mass $m$. To do so we restore the speed of light $c$ and evaluate the $p^{0}$ integral to arrive at

$$
M^{\mu_{1} \ldots \mu_{n}}=\left.\sqrt{g} \int_{\mathbb{R}^{3}} \frac{\mathrm{d}^{3} p}{(2 \pi)^{3}} \frac{p^{\mu_{1}} \ldots p^{\mu_{n}}}{\left|p_{0}\right| / c} f\right|_{p^{0}=E_{p} / c},
$$

where

$$
\frac{E_{p}}{c}=-\frac{g_{0 i} p^{i}}{g_{00}}+\sqrt{\left(\frac{g_{0 i} p^{i}}{g_{00}}\right)^{2}-\frac{p_{i} p^{i}+m^{2} c^{2}}{g_{00}}},
$$

and Latin indices only range over spatial components. In the limit $c \rightarrow \infty$, where the metric is Minkowskian, the $n$th moment involving $k$ temporal indices and $n-k$ spatial indices is

$$
\frac{1}{c^{k}} M^{0 \cdots 0 i_{1} \cdots i_{n-k}}(x) \rightarrow m^{k-1} M_{\mathrm{nr}}^{i_{1} \cdots i_{n-k}}(t, \boldsymbol{x})
$$

\footnotetext{
${ }^{4}$ The sum runs over all $2^{n}$ sets $S$ in the power set $\mathcal{P}\left(\left\{\mu_{1}, \ldots, \mu_{n}\right\}\right)$ with indices $\left(\alpha_{1}, \ldots, \alpha_{|S|}\right) \in S$ and $\left(\alpha_{|S|+1}, \ldots, \alpha_{n}\right) \in\left\{\mu_{1}, \ldots, \mu_{n}\right\} \backslash S$.

${ }^{5}$ The sum runs over all $2^{n}-1$ sets $S$ in the power set without the empty set $\mathcal{P}\left(\left\{\mu_{1}, \ldots, \mu_{n}\right\}\right) \backslash \varnothing$ with indices $\left(\alpha_{1}, \ldots, \alpha_{|S|}\right) \in S$ and $\left(\alpha_{|S|+1}, \ldots, \alpha_{n}\right) \in\left\{\mu_{1}, \ldots, \mu_{n}\right\} \backslash S$.
} 
Here $M_{\mathrm{nr}}^{i_{1} \cdots i_{n-k}}(t, \boldsymbol{x})$ is the $(n-k)$ th nonrelativistic moment and $t$ denotes time and $\boldsymbol{x}$ position in configuration space. From the nonrelativistic limit of evolution equation (17) it is then evident that the evolution of the $n$th (nonrelativistic) moment also depends on the next higher (nonrelativistic) moment. A similar argument holds true for the evolution of the nonrelativistic cumulants.

\section{Truncated cumulant expansion}

In general, the distribution function $f(x, p)$ has an infinite amount of moments, as well as cumulants, the complete set of which encode the same information. Therefore a characterization in terms of moments or cumulants are two sides of the same coin and are a matter of taste or problem at hand. However, in the collisionless case all moments of the Vlasov equation (7) are independently covariantly conserved. Truncating this hierarchy at any finite order does not generate a closed system of equations. From this point of view it does not seem possible to keep only a finite set of moments, essentially because one cannot supply enough equations of motion. Any linear redefinition of the fields does not change this. Since the cumulants are nonlinearly related to the moments and cumulants of different orders are statistically independent of each other, it seems natural to study the cumulant expansion of the distribution function in order to put forth a truncation scheme which allows to describe a finite set of independent degrees of freedom. Assuming one could truncate the expansion after the $n$th cumulant one would be left with

$\sum_{k=0}^{n}\left(\begin{array}{c}4+k-1 \\ k\end{array}\right)-\sum_{k=1}^{n}\left(\begin{array}{c}4+k-2 \\ k-1\end{array}\right)=\left(\begin{array}{c}4+n-1 \\ n\end{array}\right)$

independent degrees of freedom. Here, the first sum represents the degrees of freedom from the (fully symmetric) cumulants up to order $n$. However, these are not all independent of each other due to the on-shell constraints (12) which reduce the independent degrees of freedom. This is represented by the second sum.

From the full set of cumulants one can in principle reconstruct the distribution function $\tilde{f}(x, p)$. However, this is not so for a finite truncation, with the exceptions of a truncation after the first or second cumulant, corresponding to a degenerate or normal distribution, respectively.

To work out the consequences of such truncations in more detail it is useful to decompose the particle number current as

$$
N^{\mu}=n u^{\mu}+\nu^{\mu},
$$

and the energy-momentum tensor as

$$
T^{\mu \nu}=\epsilon u^{\mu} u^{\nu}+\left(p+\pi_{\text {bulk }}\right) \Delta^{\mu \nu}+\pi^{\mu \nu}+2 q^{(\mu} u^{\nu)} .
$$

Here $n$ is the particle number density, $u^{\mu}$ is the local fluid four-velocity normalized to $u_{\mu} u^{\mu}=-1$ and $\nu^{\mu}$ is the diffusion current orthogonal to the fluid velocity, $u_{\mu} \nu^{\mu}=0$. Further, $\epsilon$ is the energy density in the local rest frame, $p$ the thermodynamic pressure which is related to $n$ and $\epsilon$ by the equilibrium expression, $\pi_{\text {bulk }}$ is the bulk viscous pressure, $\Delta^{\mu \nu}=u^{\mu} u^{\nu}+g^{\mu \nu}$ is a projector orthogonal to the fluid velocity, $\pi^{\mu \nu}$ is the shear stress tensor, which is symmetric, transverse to the fluid velocity $u_{\mu} \pi^{\mu \nu}=0$ and traceless $\pi_{\mu}{ }^{\mu}=0$ and $q^{\mu}$ is the heat current which is orthogonal to the fluid velocity, $u_{\mu} q^{\mu}=0$. In the following we abbreviate the sum of thermodynamic and bulk viscous pressure as an effective pressure, $p_{\text {eff }}=p+\pi_{\text {bulk }}$. These rather general relations can be specialized to a frame by fixing the fluid four-velocity. Common choices are the Landau frame [9], where the fluid four-velocity is a timelike eigenvector of the energy-momentum tensor and the heat current vanishes, or the Eckart frame [8], in which the fluid four-velocity is defined by the direction of the particle number current so that one has vanishing diffusion current.

In the most simple nontrivial case, one truncates the cumulant expansion after the first order and obtains the socalled single-stream approximation. It is characterized by four independent degrees of freedom and for a single particle species with unique mass $m$ the on-shell constraint (13) leads to $z=n / m$. The modified distribution function is a degenerate distribution,

$$
\tilde{f}=\frac{(2 \pi)^{4}}{\sqrt{g}} \frac{n}{m} \delta^{(4)}(p-m u),
$$

where $\delta^{(4)}$ denotes the four-dimensional Dirac delta function. In this case one has $\epsilon=n m$ and $p_{\text {eff }}=\nu^{\mu}=$ $q^{\mu}=\pi^{\mu \nu}=0$, or in other words, the particle number current and the energy-momentum tensor are the ones of a perfect pressureless fluid.

Including the second cumulant and truncating the expansion at third order, one obtains a normal distribution characterized by ten independent degrees of freedom. ${ }^{6}$ Again specializing to a single particle species with unique mass $m$, the second moment on-shell constraint $g_{\mu \nu} T^{\mu \nu}=$ $m^{2} z$ leads to

$$
z=\frac{\epsilon-3 p_{\mathrm{eff}}}{m^{2}}
$$

\footnotetext{
${ }^{6} \mathrm{~A}$ general (unnormalized) four-dimensional normal distribution is as usual characterized by cumulants up to second order, corresponding to 15 degrees of freedom. However, the on-shell constraints (12) reduce the independent degrees of freedom to ten, see also Eq. (23) and the discussion thereafter.
} 
The third moment on-shell constraint projected along the fluid velocity $u_{\rho} g_{\mu \nu} M^{\mu \nu \rho}=m^{2} u_{\rho} N^{\rho}$ gives

$$
\frac{2}{z^{3}}\left[n^{3}-z n \epsilon-n \nu_{\mu} \nu^{\mu}+z q_{\mu} \nu^{\mu}\right]=g_{\mu \nu} C^{\mu \nu \rho} u_{\rho}=0,
$$

while the projection orthogonal to the fluid velocity $\Delta^{\mu}{ }_{\sigma} g_{\nu \rho} M^{\nu \rho \sigma}=m^{2} \Delta^{\mu}{ }_{\sigma} N^{\sigma}$ yields

$$
\begin{aligned}
& \frac{2}{z^{3}}\left[\left(\nu_{\nu} \nu^{\nu}-n^{2}-z p_{\text {eff }}\right) \nu^{\mu}-z \pi^{\mu \nu} \nu_{\nu}+z n q^{\mu}\right] \\
& =\Delta^{\mu}{ }_{\sigma} g_{\nu \rho} C^{\nu \rho \sigma}=0 .
\end{aligned}
$$

The modified distribution function is given by

$$
\begin{aligned}
\tilde{f}= & \frac{(2 \pi)^{4}}{\sqrt{g}} \frac{n^{2}}{\epsilon} \delta\left(u \cdot p-\frac{\epsilon}{n}\right) \frac{1}{(2 \pi)^{\frac{3}{2}} \operatorname{det}\left(C_{\alpha \beta}\right)^{\frac{1}{2}}} \\
& \times \exp \left\{-\frac{1}{2} p^{\mu}\left(C^{-1}\right)_{\mu \nu} p^{\nu}\right\}
\end{aligned}
$$

where $u \cdot p=u_{\mu} p^{\mu}$ and due to the constraint (29) the second cumulant is purely transverse to the fluid velocity,

$$
C^{\mu \nu}=p_{\text {eff }} \Delta^{\mu \nu}+\pi^{\mu \nu},
$$

and therefore the parallel part collapses to a Dirac delta function. The constraints (27) and (28) can be combined to give

$$
\frac{n^{2}}{\epsilon}=\frac{\epsilon-3 p_{\mathrm{eff}}}{m^{2}},
$$

reducing the independent degrees of freedom to ten. Interestingly enough this truncation implies vanishing diffusion and heat current and thus the Landau and Eckart frame are the same. Formally we now have a fluid with nonvanishing bulk viscous pressure and shear stress.

This truncation scheme can be straightforwardly generalized to higher orders. However, there are no distributions which are characterized by a finite number of cumulants beside the degenerate and normal distributions and one is therefore not able to explicitly reconstruct $\tilde{f}(x, p)$. Further one cannot find a corresponding distribution function $f(x, p)$ because one is not able reconstruct the $\delta\left(p^{2}+m^{2}\right)$. Nevertheless, as an approximate model for a more complex form, Eq. (30) may be quite reasonable. A nice feature is that the cumulants that govern it have rather transparent equations of motion that can be solved in the spirit of a nonideal fluid approximation as we discuss in the next section.

While we are in principle able to truncate the cumulant expansion of the distribution function, the question arises whether these truncations are preserved by the Vlasov equation (18). Interestingly enough the single-stream approximation (26) is (apparently) preserved since no higher order cumulants are sourced by terms solely depending on the first cumulant. However, this apparent selfconsistency is not stable under perturbations, that is as soon as any other cumulant obtains a nonvanishing value, cumulants of all orders are generated. This also implies that any truncation beyond the single-stream approximation is not preserved by the Vlasov equation and higher order cumulants are generated throughout evolution in time.

Qualitatively this can be seen by explicitly constructing the first few equations of motion from Eq. (19),

$$
\begin{gathered}
C^{\nu} \nabla_{\nu} C^{\mu}+\nabla_{\nu} C^{\nu \mu}+C^{\mu \nu} \nabla_{\nu} \ln (z)=0 \\
C^{\rho} \nabla_{\rho} C^{\mu \nu}+\nabla_{\rho} C^{\rho \mu \nu}+2 C^{(\mu \mid \rho} \nabla_{\rho} C^{\mid \nu)}+C^{\mu \nu \rho} \nabla_{\rho} \ln (z)=0 \\
C^{\sigma} \nabla_{\sigma} C^{\mu \nu \rho}+\nabla_{\sigma} C^{\sigma \mu \nu \rho}+3 C^{(\mu \mid \sigma} \nabla_{\sigma} C^{\mid \nu \rho)} \\
+3 C^{(\mu \nu \mid \sigma} \nabla_{\sigma} C^{\mid \rho)}+C^{\mu \nu \rho \sigma} \nabla_{\sigma} \ln (z)=0
\end{gathered}
$$

where the vertical bars denote exclusion of the symmetrization of indices. Since there are no terms in Eq. (34) or (35) that solely depend on the first order cumulant it is within the single-stream approximation not possible to generate the second or third order cumulant from evolution by the Vlasov equation alone. On the other hand, there are nonlinear terms in Eq. (35) involving only the second order cumulant. That is, even if the third order cumulant is initially absent it is dynamically generated given a nonvanishing second order cumulant. Similar arguments hold true for all higher order cumulants as can be checked from the combinations of cumulants appearing in the quadratic terms of Eq. (19). This prevents any truncation apart from the single-stream approximation to be preserved.

However, the self-consistency of the single-stream approximation is only apparent due to the phenomenon of shell-crossing, when multiple streams of matter coexist at the same region in space. At this point in configuration space the velocity field in Eq. (26) is multivalued and the corresponding distribution function $\tilde{f}(x, p)$ has nonvanishing second and higher order cumulants [50]. To go beyond the shell-crossing regime one therefore needs a description which is not limited to the first order cumulant only.

\section{CLOSED SYSTEM OF EQUATIONS}

\section{A. Evolution equations}

The particle number density and energy-momentum tensor have 14 independent degrees of freedom which we parametrize in terms of the fields introduced in Eqs. (24) and (25). Evolution equations for these can be obtained from the covariant conservation of the first three moments, 


$$
\nabla_{\mu} N^{\mu}=0, \quad \nabla_{\nu} T^{\mu \nu}=0, \quad \nabla_{\rho} M^{\mu \nu \rho}=0,
$$

by expressing the third moment in terms of lower order moments and the third cumulant, as done in Eq. (16).

The conservation of the particle number current yields an evolution equation for the particle number density,

$$
u^{\mu} \nabla_{\mu} n+n \nabla_{\mu} u^{\mu}+\nabla_{\mu} \nu^{\mu}=0 .
$$

Projecting the conservation of the energy-momentum tensor along the fluid four-velocity, $u_{\mu} \nabla_{\nu} T^{\mu \nu}=0$, gives an evolution equation for the energy density,

$$
u^{\mu} \nabla_{\mu} \epsilon+\left(\epsilon+p_{\mathrm{eff}}\right) \nabla_{\mu} u^{\mu}+\pi^{\mu \nu} \nabla_{\mu} u_{\nu}+\nabla_{\mu} q^{\mu}+q^{\nu} u^{\mu} \nabla_{\mu} u_{\nu}=0
$$

while a projection orthogonal, $\Delta^{\mu}{ }_{\nu} \nabla_{\rho} T^{\nu \rho}=0$, yields essentially an evolution equation of the fluid four-velocity,

$$
\left(\epsilon+p_{\mathrm{eff}}\right) u^{\nu} \nabla_{\nu} u^{\mu}+\Delta^{\mu \nu} \nabla_{\nu} p_{\mathrm{eff}}+\Delta^{\mu}{ }_{\nu} \nabla_{\rho} \pi^{\rho \nu}+\Delta^{\mu}{ }_{\nu} u^{\rho} \nabla_{\rho} q^{\nu}+q^{\mu} \nabla_{\nu} u^{\nu}+q^{\nu} \nabla_{\nu} u^{\mu}=0
$$

A projection onto the orthogonal parts of the covariant conservation of the third moment, $\Delta_{\mu \nu} \nabla_{\rho} M^{\mu \nu \rho}=0$, gives an evolution equation for the effective pressure,

$$
\begin{aligned}
\left(n u^{\rho}\right. & \left.+\nu^{\rho}\right)\left\{3 \nabla_{\rho} p_{\mathrm{eff}}+2 q_{\mu} \nabla_{\rho} u^{\mu}+\left(4 \nu_{\mu} \nu^{\mu}-3 z p_{\mathrm{eff}}\right) \frac{\nabla_{\rho} z}{z^{2}}-\frac{4}{z}\left(n \nu_{\mu} \nabla_{\rho} u^{\mu}+\nu_{\mu} \nabla_{\rho} \nu^{\mu}\right)\right\} \\
& -2\left(p_{\mathrm{eff}} \nu^{\rho}+\nu_{\mu} \pi^{\mu \rho}+q_{\mu} \nu^{\mu} u^{\rho}\right) \frac{\nabla_{\rho} z}{z}+2\left(p_{\mathrm{eff}} \Delta^{\mu \rho}+\pi^{\mu \rho}+q^{\mu} u^{\rho}\right)\left\{n \nabla_{\rho} u_{\mu}+\nabla_{\rho} \nu_{\mu}\right\} \\
& =-z \Delta_{\mu \nu}\left\{z \nabla_{\rho} C^{\mu \nu \rho}+C^{\mu \nu \rho} \nabla_{\rho} z\right\} .
\end{aligned}
$$

The evolution equation for the three degrees of freedom parametrized by the diffusion current $\nu^{\mu}$ and heat current $q^{\mu}$ is obtained from the projection $\Delta^{\rho}{ }_{\mu} u_{\nu} \nabla_{\rho} M^{\mu \nu \rho}=0$,

$$
\begin{aligned}
\left(n u^{\rho}\right. & \left.+\nu^{\rho}\right)\left\{\Delta^{\rho}{ }_{\mu} \nabla_{\rho} q^{\mu}+\left(\epsilon+p_{\text {eff }}\right) \nabla_{\rho} u^{\alpha}+\pi^{\alpha \mu} \nabla_{\rho} u_{\nu}+\left(4 n \nu^{\alpha}-z q^{\alpha}\right) \frac{\nabla_{\rho} z}{z^{2}}\right. \\
& \left.-\frac{2}{z}\left(n^{2} \nabla_{\rho} u^{\alpha}+\Delta^{\alpha}{ }_{\mu} n \nabla_{\rho} \nu^{\mu}+\nu^{\alpha} \nabla_{\rho} n+\nu^{\alpha} \nu^{\nu} \nabla_{\rho} u_{\nu}\right)\right\} \\
& +\left(\epsilon u^{\rho}+q^{\rho}\right)\left\{\Delta^{\alpha}{ }_{\mu} \nabla_{\rho} \nu^{\mu}+n \nabla_{\rho} u^{\alpha}-\nu^{\alpha} \frac{\nabla_{\rho} z}{z}\right\}+\left(p_{\text {eff }} \Delta^{\rho \alpha}+\pi^{\rho \alpha}+u^{\rho} q^{\alpha}\right)\left\{\nabla_{\rho} n+\nu^{\nu} \nabla_{\rho} u_{\nu}-n \frac{\nabla_{\rho} z}{z}\right\} \\
& =z \Delta^{\rho}{ }_{\mu} u_{\nu}\left\{z \nabla_{\rho} C^{\mu \nu \rho}+C^{\mu \nu \rho} \nabla_{\rho} z\right\} .
\end{aligned}
$$

Finally, the evolution for the shear stress tensor $\pi^{\mu \nu}$ follows from the projection $P^{\alpha \beta}{ }_{\mu \nu} \nabla_{\rho} M^{\mu \nu \rho}=0$,

$$
\begin{aligned}
\left(n u^{\rho}\right. & \left.+\nu^{\rho}\right) P_{\mu \nu}^{\alpha \beta}\left\{\nabla_{\rho} \pi^{\mu \nu}+2 q^{\nu} \nabla_{\rho} u^{\mu}+\left(4 \nu^{\mu} \nu^{\nu}-z \pi^{\mu \nu}\right) \frac{\nabla_{\rho} z}{z^{2}}\right\}+2\left(p_{\mathrm{eff}} \Delta^{\mu \rho}+\pi^{\mu \rho}+q^{\mu} u^{\rho}\right) P^{\alpha \beta} \quad\left\{n \nabla_{\rho} u^{\nu}+\nabla_{\rho} \nu^{\nu}-\nu^{\nu} \frac{\nabla_{\rho} z}{z}\right\} \\
& =-z P^{\alpha \beta}{ }_{\mu \nu}\left\{z \nabla_{\rho} C^{\mu \nu \rho}+C^{\mu \nu \rho} \nabla_{\rho} z\right\},
\end{aligned}
$$

where the symmetric, traceless and transverse projector reads

$$
P^{\alpha \beta}{ }_{\mu \nu}=\frac{1}{2} \Delta^{\alpha}{ }_{\mu} \Delta^{\beta}{ }_{\nu}+\frac{1}{2} \Delta^{\alpha}{ }_{\nu} \Delta^{\beta}{ }_{\mu}-\frac{1}{3} \Delta^{\alpha \beta} \Delta_{\mu \nu}
$$

It is evident that the system of Eqs. (37)-(42) is not closed, since they couple to the normalization $z$ and the third cumulant $C^{\mu \nu \rho}$. In order to close the system of equations we impose a truncation of the cumulant expansion after the second cumulant, $C^{\mu \nu \rho}=0$, and use the energy-momentum tensor on-shell constraint (27) to eliminate $z$. Strictly speaking not all of the equations are independent of each other due to Eqs. (28) and (29). These reduce the independent degrees of freedom to ten in accordance with formula (23). Nevertheless, we assume that these constraints can be neglected with the reasoning as follows. We know from the discussion at the end of Sec. II that 
the imposed truncation is not preserved by the Vlasov equation and the third cumulant is generated throughout the evolution in time. Therefore the constraints (28) and (29) are not strictly satisfied but rather proportional to the third cumulant as indicated on the right-hand side. That is, we do not assume the third cumulant to be exactly zero throughout time evolution, but rather to stay small enough to be neglected in the evolution equations.

The motivation for this truncation is partly derived from the nonrelativistic limit, where the single-stream approximation is frequently used to model the evolution of cold dark matter. A similar truncation of the cumulant expansion after the second order has been used to model dark matter with nonvanishing velocity dispersion which at least cures the break-down of the description at shell crossing $[52,53]$.

Furthermore, for a (sufficiently cold) dark matter candidate which thermally decouples in the early universe, one expects the momentum to be initially normal distributed, while higher order cumulants are negligible. As a consequence of gravitational dynamics the distribution function is pushed away from local thermal equilibrium and local thermalization times can be arbitrarily large. This makes it hard to justify a derivative expansion which ultimately relies on short thermalization times.

Finally, from a heuristic point of view, we argue that the first and second order cumulants are more relevant than higher order cumulants for gravitational dynamics since they enter directly in the Einstein field equations.

Collecting the 14 degrees of freedom in a superfield $\Phi^{a}(x)$ the evolution equations (37)-(42) can be written in the quasilinear form

$$
\mathcal{A}_{a} u^{\rho} \nabla_{\rho} \Phi^{a}+\mathcal{B}^{\rho}{ }_{a} \nabla_{\rho} \Phi^{a}=0
$$

Here $\mathcal{A}_{a}$ and $\mathcal{B}^{\rho}{ }_{a}$ are field dependent matrices and $u_{\rho} \mathcal{B}^{\rho}{ }_{a}=0$. The index $a$ carries the appropriate amount of covariant indices, e.g., in the Eckart frame one has $\Phi^{a}=\left(n, \epsilon, p_{\text {eff }}, u^{\mu}, q^{\mu}, \pi^{\mu \nu}\right)$. Since the expressions for the matrices $\mathcal{A}_{a}$ and $\mathcal{B}^{\rho}{ }_{a}$ are less transparent and rather cumbersome if written explicitly, we restrain from displaying them. We checked that the matrix $\mathcal{A}_{a}$ is diagonalizable and invertible, making the system hyperbolic [54]. Finally, we are left with a closed, covariant and hyperbolic system of equations which describe the 14 degrees of freedom introduced in Eqs. (24) and (25).

\section{B. FLRW cosmology}

As a simple cosmological working example we consider a spatially flat Friedmann-Lemaitre-Robertson-Walker metric with line element

$$
\mathrm{d} s^{2}=-\mathrm{d} t^{2}+a(t)^{2} \delta_{i j} \mathrm{~d} x^{i} \mathrm{~d} x^{j}
$$

where $a(t)$ is the dimensionless scale factor which parametrizes the relative spatial expansion of the Universe. Due to the symmetries of the metric, namely homogeneity and isotropy, the particle number current and energymomentum tensor have the form of a perfect fluid,

$$
N^{\mu}=n u^{\mu}, \quad T^{\mu \nu}=\epsilon u^{\mu} u^{\nu}+p_{\mathrm{eff}} \Delta^{\mu \nu} .
$$

Here the energy density $\epsilon(t)$ and effective pressure $p_{\text {eff }}(t)$ are functions of time only and the four-velocity is given by $u^{\mu}=(1,0,0,0)$. We define the effective equation of state parameter as the ratio of the effective pressure and energy density, $\omega_{\text {eff }}=p_{\text {eff }} / \epsilon$. The evolution equations (37) and (38) read

$$
\partial_{t} n+3 H n=0, \quad \partial_{t} \epsilon+3 H\left(1+\omega_{\mathrm{eff}}\right) \epsilon=0,
$$

while Eq. (40) gives

$$
\partial_{t} \omega_{\text {eff }}+2 H\left(1-3 \omega_{\text {eff }}\right) \omega_{\text {eff }}=0,
$$

and $H=\partial_{t} a / a$ is the Hubble rate. These equations can be solved in terms of the scale factor,

$$
\begin{aligned}
n & =n_{0} a^{-3}, \\
\epsilon & =\epsilon_{0} a^{-4}\left[a^{2}\left(1-3 \omega_{\mathrm{eff}, 0}\right)+3 \omega_{\mathrm{eff}, 0}\right]^{\frac{1}{2}}, \\
\omega_{\mathrm{eff}} & =\omega_{\mathrm{eff}, 0}\left[a^{2}\left(1-3 \omega_{\mathrm{eff}, 0}\right)+3 \omega_{\mathrm{eff}, 0}\right]^{-1},
\end{aligned}
$$

where quantities subscripted with a 0 are the values at $a(t)=1$, corresponding to today. The particle number density decays as expected with the expansion of space while the decay of the energy density also depends on the effective equation of state parameter. Figure 1 displays the growth of the effective equation of state parameter as a function of itself. The arrows indicate the flow of the

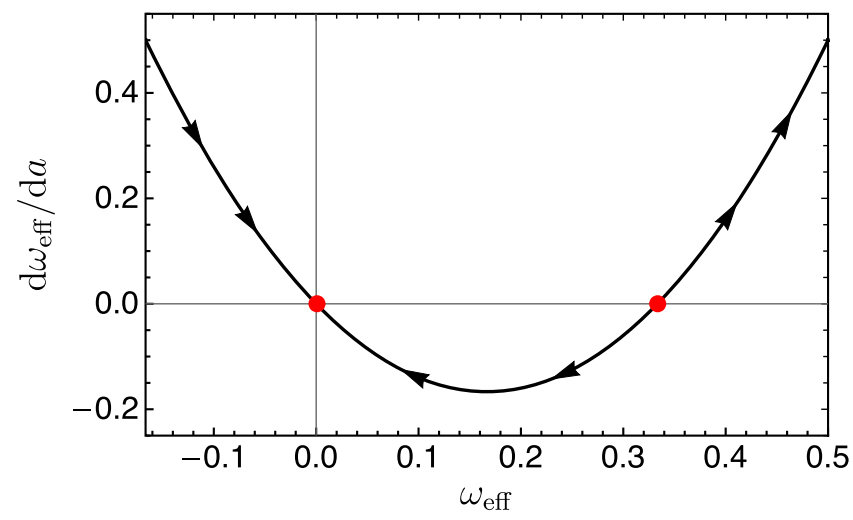

FIG. 1. The flow of the effective equation of state $\omega_{\text {eff }}$ is indicated by arrows and the fix points as red dots. It has an attractive fixed point at $\omega_{\text {eff }}=0$ corresponding to nonrelativistic matter as well as a repulsive fixed point at $\omega_{\text {eff }}=1 / 3$ corresponding to ultrarelativistic radiation. 
solution and the red dots indicate the fixed points. There is an attractive fixed point at $\omega_{\text {eff }}=0$ corresponding to nonrelativistic matter and a repulsive fixed point at $\omega_{\text {eff }}=$ $1 / 3$ corresponding to ultrarelativistic radiation. For an effective equation of state parameter $\omega_{\text {eff }}<1 / 3$ the solution evolves towards the attractive nonrelativistic solution fixed point as is expected for matter that is noninteracting except for gravity. For an effective equation of state parameter $\omega_{\text {eff }}>1 / 3$ the solution exhibits a strong growth, but the physical interpretation behind such values of the equation of state parameter is not clear.

In a next step it would be interesting to treat nonhomogeneous solutions and cosmological structure formation. The full set of nonideal fluid fields, including the peculiar fluid velocity, shear stress and diffusion or heat current, are then expected to become nontrivial. Interestingly, perturbations in these fields can also influence the overall cosmological expansion through a dissipative backreaction [55].

\section{CONCLUSIONS}

Starting from a general relativistic kinetic theory approach for a system of collisionless classical point particles we presented the method of moments and cumulants of the one-particle phase-space distribution function. We studied how the moments and cumulants evolve under the relativistic Vlasov equation and found in particular that all moments are covariantly conserved while the cumulants follow a more complex nonlinear evolution equation. For the first and second moment these are the common covariant conservation laws related to the particle number current and energy-momentum tensor, but the evolution of higher order moments furnish additional evolution equations.

We showed how the cumulant expansion of the distribution function can be truncated at finite order and explicitly performed this truncation after the first and second cumulant, corresponding to the single-stream and a Gaussian approximation, respectively. In particular the Gaussian approximation is capable of describing a nonideal fluid with nonvanishing bulk viscous pressure and shear stress. We discussed that these kinds of truncations are not preserved by the Vlasov equation since higher order cumulants are naturally generated by lower order cumulants.

From the covariant conservation of the first three moments we derived a closed, covariant and hyperbolic system of equations by neglecting the third cumulant. The equations give the evolution of the 14 degrees of freedom of the particle number current and energy-momentum tensor and can be used to describe a general relativistic nonideal fluid. As a working example we considered a FriedmannLemaître-Robertson-Walker cosmology with nonvanishing dynamic pressure and solve its time evolution. We find that the solution has an attractive and repulsive fixed point, corresponding to nonrelativistic matter and ultrarelativistic radiation, respectively.

The equations of motion for a nonideal fluid approximation to dark matter that we have derived can be extended in different directions. In a next step, one could expand the third order cumulant in a derivative expansion with respect to the fluid velocity, which may be more appropriate for the collisional case discussed in the Appendix. Further one could include dark matter self-interactions or interactions with baryonic matter. Another would be to include quantum effects. Moreover, it would of course be highly interesting to solve the evolution equations for the nonideal fluid fields or cumulants directly, either numerically or with further analytical techniques such as perturbation theory or field theoretic methods similar to those developed in Refs. [56-58]. In particular we are curious to see whether the truncated cumulant expansion developed here, or an extension of it, can agree with numerical solutions of the Vlasov equation through $N$-body simulations. This might lead to a rather useful framework to study extensions of the collisionless cold dark matter model and for a comparison to observational data.

\section{ACKNOWLEDGMENTS}

The authors thank E. Grossi for useful discussions. This work is supported by the Deutsche Forschungsgemeinschaft (German Research Foundation) under Germany's Excellence Strategy and the Cluster of Excellence EXC 2181 (STRUCTURES), the Collaborative Research Centre SFB 1225 (ISOQUANT) as well as Research Grant FL 736/3-1.

\section{APPENDIX: RELAXATION-TIME APPROXIMATION}

In a setting with collisions, as for example for selfinteracting dark matter, the evolution of the distribution function is determined by the relativistic Boltzmann equation $[48,49]$,

$$
\left[p^{\mu} \frac{\partial}{\partial x^{\mu}}-\Gamma_{\rho \sigma}^{\mu} p^{\rho} p^{\sigma} \frac{\partial}{\partial p^{\mu}}\right] f=\mathcal{C}[f]
$$

which differs from the Vlasov equation (7) by the collision integral $C[f]$, which in general is a nonlinear functional of the distribution function. The situation is greatly simplified in the relaxation-time approximation $[59,60]$, for which the collision term is

$$
\mathcal{C}=-(u \cdot p) \frac{f-f_{\mathrm{eq}}}{\tau_{\mathrm{eq}}}
$$

Here $f_{\text {eq }}(x, p)$ is the equilibrium distribution function and $\tau_{\mathrm{eq}}$ the relaxation time. Defining the equilibrium moments $M_{\mathrm{eq}}^{\mu_{1} \cdots \mu_{n}}(x)$ in the same manner as done in Eq. (10) for the 
equilibrium distribution function, one can derive the evolution equation

$$
\nabla_{\nu} M^{\nu \mu_{1} \cdots \mu_{n}}=-\frac{u_{\nu}}{\tau_{\mathrm{eq}}}\left[M^{\nu \mu_{1} \cdots \mu_{n}}-M_{\mathrm{eq}}^{\nu \mu_{1} \cdots \mu_{n}}\right]
$$

While the moments are no longer covariantly conserved, they are still independent of each other at each order. Since the particle number current and energy-momentum tensor are still covariantly conserved, we can infer their equilibrium form,

$$
N_{\mathrm{eq}}^{\mu}=n u^{\mu}, \quad T_{\mathrm{eq}}^{\mu \nu}=\epsilon u^{\mu} u^{\nu}+p \Delta^{\mu \nu} .
$$

This also fixes the equilibrium normalization to $z_{\mathrm{eq}}=(\epsilon-3 p) / \mathrm{m}^{2}$. Proceeding in the same manner as done in Sec. III to derive Eqs. (37)-(42), Eqs. (37)-(39) are unchanged due to the covariant conservation of the particle number current and energy-momentum tensor. However, Eqs. (40)-(42) obtain additional contributions due to the terms on the right-hand side of Eq. (A3) stemming from the relaxation-time approximation of the collision integral. The additional terms appearing on the right-hand sides of Eqs. (40)-(42) are

$$
\begin{aligned}
& -\frac{1}{\tau_{\mathrm{eq}}}\left[3 n\left(\frac{z}{z_{\mathrm{eq}}} p-p_{\mathrm{eff}}\right)+2\left(\frac{n}{z} \nu_{\mu}-q_{\mu}\right) \nu^{\mu}\right. \\
& \left.\quad+z \Delta_{\mu \nu} u_{\rho}\left(z C^{\mu \nu \rho}-z_{\mathrm{eq}} C_{\mathrm{eq}}^{\mu \nu \rho}\right)\right], \\
& -\frac{1}{\tau_{\mathrm{eq}}}\left[\left(\frac{2 n}{z} n-\epsilon\right) \nu^{\alpha}-2 n q^{\alpha}-z \Delta^{\alpha}{ }_{\mu} u_{\nu} u_{\rho}\left(z C^{\mu \nu \rho}-z_{\mathrm{eq}} C_{\mathrm{eq}}^{\mu \nu \rho}\right)\right], \\
& \quad-\frac{1}{\tau_{\mathrm{eq}}}\left[2 P^{\alpha \beta}{ }_{\mu \nu}\left(\frac{n}{z} \nu^{\mu}-q^{\mu}\right) \nu^{\nu}-n \pi^{\alpha \beta}+z P^{\alpha \beta}{ }_{\mu \nu} u_{\rho}\left(z C^{\mu \nu \rho}-z_{\mathrm{eq}} C_{\mathrm{eq}}^{\mu \nu \rho}\right)\right], \\
& \text { respectively. This approach may be generalized to more } \\
& \text { complex collision integrals, such as the Stoßzahlansatz, } \\
& \text { although it is not clear whether one can explicitly solve the } \\
& \text { integral in this case. For the Gaussian approximation } \\
& \text { employed in this work, one could hope to have a chance } \\
& \text { at solving the collision integral, although it heavily depends } \\
& \text { on the explicit form of the collision kernel. }
\end{aligned}
$$

[1] E. W. Kolb and M. S. Turner, The Early Universe (AddisonWesley, Redwood City, CA, 1990).

[2] S. Weinberg, Cosmology (Oxford University Press, Oxford, 2008).

[3] P. J. E. Peebles, The Large-Scale Structure of the Universe (Princeton University Press, Princeton, NJ, 1980).

[4] T. Schäfer and D. Teaney, Nearly perfect fluidity: From cold atomic gases to hot quark gluon plasmas, Rep. Prog. Phys. 72, 126001 (2009).

[5] U. Heinz and R. Snellings, Collective flow and viscosity in relativistic heavy-ion collisions, Annu. Rev. Nucl. Part. Sci. 63, 123 (2013).

[6] C. Gale, S. Jeon, and B. Schenke, Hydrodynamic modeling of heavy-ion collisions, Int. J. Mod. Phys. A 28, 1340011 (2013).

[7] R. D. de Souza, T. Koide, and T. Kodama, Hydrodynamic approaches in relativistic heavy ion reactions, Prog. Part. Nucl. Phys. 86, 35 (2016).

[8] C. Eckart, The thermodynamics of irreversible processes. III. Relativistic theory of the simple fluid, Phys. Rev. 58, 919 (1940).

[9] L.D. Landau and E. M. Lifschitz, Fluid Mechanics (Pergamon Press, Oxford, 1959).

[10] G. Pichon, Étude relativiste de fluides visqueux et chargés, Ann. Inst. Henri Poincaré A 2, 21 (1965).

[11] W. A. Hiscock and L. Lindblom, Stability and causality in dissipative relativistic fluids, Ann. Phys. (N.Y.) 151, 466 (1983).
[12] W. A. Hiscock and L. Lindblom, Generic instabilities in first-order dissipative relativistic fluid theories, Phys. Rev. D 31, 725 (1985).

[13] W. A. Hiscock and L. Lindblom, Linear plane waves in dissipative relativistic fluids, Phys. Rev. D 35, 3723 (1987).

[14] A. Muronga, Causal theories of dissipative relativistic fluid dynamics for nuclear collisions, Phys. Rev. C 69, 034903 (2004).

[15] F. S. Bemfica, M. M. Disconzi, and J. Noronha, Causality and existence of solutions of relativistic viscous fluid dynamics with gravity, Phys. Rev. D 98, 104064 (2018).

[16] P. Kovtun, First-order relativistic hydrodynamics is stable, J. High Energy Phys. 10 (2019) 034.

[17] F. S. Bemfica, M. M. Disconzi, and J. Noronha, Nonlinear causality of general first-order relativistic viscous hydrodynamics, Phys. Rev. D 100, 104020 (2019).

[18] R. E. Hoult and P. Kovtun, Stable and causal relativistic Navier-Stokes equations, J. High Energy Phys. 06 (2020) 067.

[19] H. Grad, On the kinetic theory of rarefied gases, Commun. Pure Appl. Math. 2, 331 (1949).

[20] I. Müller, Zum Paradoxon der Wärmeleitungstheorie, Z. Phys. 198, 329 (1967).

[21] W. Isreal, Nonstationary irreversible thermodynamics: A causal relativistic theory, Ann. Phys. (N.Y.) 100, 310 (1976).

[22] W. Isreal and J. M. Stewart, Transient relativistic thermodynamics and kinetic theory, Ann. Phys. (N.Y.) 118, 341 (1979). 
[23] R. Baier, P. Romatschke, D. T. Son, A. O. Starinets, and M. A. Stephanov, Relativistic viscous hydrodynamics, conformal invariance, and holography, J. High Energy Phys. 04 (2008) 100.

[24] G. S. Denicol, H. Niemi, E. Molnár, and D. H. Rischke, Derivation of transient relativistic fluid dynamics from the Boltzmann equation, Phys. Rev. D 85, 114047 (2012); 91, 039902(E) (2015).

[25] G. S. Denicol, E. Molnár, H. Niemi, and D. H. Rischke, Derivation of fluid dynamics from kinetic theory with the 14-moment approximation, Eur. Phys. J. A 48, 170 (2012).

[26] G. S. Denicol, X.-G. Huang, E. Molnár, G. M. Monteiro, H. Niemi, J. Noronha, D. H. Rischke, and Q. Wang, Nonresistive dissipative magnetohydrodynamics from the Boltzmann equation in the 14-moment approximation, Phys. Rev. D 98, 076009 (2018).

[27] G. S. Denicol, E. Molnár, H. Niemi, and D. H. Rischke, Resistive dissipative magnetohydrodynamics from the Boltzmann-Vlasov equation, Phys. Rev. D 99, 056017 (2019).

[28] A. Kurkela, U. A. Wiedemann, and B. Wu, Nearly isentropic flow at sizable $\eta / s$, Phys. Lett. B 783, 274 (2018).

[29] A. Kurkela, U. A. Wiedemann, and B. Wu, Opacity dependence of elliptic flow in kinetic theory, Eur. Phys. J. C 79, 759 (2019).

[30] J.-P. Blaizot and L. Yan, Fluid dynamics of out of equilibrium boost invariant plasmas, Phys. Lett. B 780, 283 (2018).

[31] J.-P. Blaizot and L. Yan, Emergence of hydrodynamical behavior in expanding ultrarelativistic plasmas, Ann. Phys. (Amsterdam) 412, 167993 (2020).

[32] C. S. Gallagher and T. Clifton, Relativistic Euler equations in cosmologies with nonlinear structures, Phys. Rev. D 98, 103516 (2018).

[33] L. Castiblanco, R. Gannouji, J. Noreña, and C. Stahl, Relativistic cosmological large scale structures at one-loop, J. Cosmol. Astropart. Phys. 07 (2019) 030.

[34] R. Maartens, Dissipative cosmology, Classical Quantum Gravity 12, 1455 (1995).

[35] W. Zimdahl, Bulk viscous cosmology, Phys. Rev. D 53, 5483 (1996).

[36] F. S. Bemfica, M. M. Disconzi, and J. Noronha, Causality of the Einstein-Israel-Stewart Theory with Bulk Viscosity, Phys. Rev. Lett. 122, 221602 (2019).

[37] H. Andréasson, The Einstein-Vlasov system/kinetic theory, Living Rev. Relativity 14, 4 (2011).

[38] O. F. Piattella, D. C. Rodrigues, J. C. Fabris, and J. A. de Freitas Pacheco, Evolution of the phase-space density and the Jeans scale for dark matter derived from the VlasovEinstein equation, J. Cosmol. Astropart. Phys. 11 (2013) 002.

[39] O. F. Piattella, L. Casarini, J. C. Fabris, and J. A. de Freitas Pacheco, Dark matter velocity dispersion effects on CMB and matter power spectra, J. Cosmol. Astropart. Phys. 02 (2016) 024.

[40] D. Baumann, A. Nicolis, L. Senatore, and M. Zaldarriaga, Cosmological non-linearities as an effective fluid, J. Cosmol. Astropart. Phys. 07 (2012) 051.

[41] J. J. M. Carrasco, M. P. Hertzberg, and L. Senatore, The effective field theory of cosmological large scale structures, J. High Energy Phys. 09 (2012) 082.
[42] R. A. Porto, L. Senatore, and M. Zaldarriaga, The Lagrangianspace effective field theory of large scale structures, J. Cosmol. Astropart. Phys. 05 (2014) 022.

[43] C. Viermann, F. Fabis, E. Kozlikin, R. Lilow, and M. Bartelmann, Nonequilibrium statistical field theory for classical particles: Basic kinetic theory, Phys. Rev. E 91, 062120 (2015).

[44] M. Bartelmann, F. Fabis, D. Berg, E. Kozlikin, R. Lilow, and C. Viermann, A microscopic, nonequilibrium, statistical field theory for cosmic structure formation, New J. Phys. 18, 043020 (2016).

[45] M. Bartelmann et al., Cosmic structure formation with kinetic field theory, Ann. Phys. (Berlin) 531, 1800446 (2019).

[46] P. Friedrich and T. Prokopec, Scalar field dark matter in hybrid approach, Phys. Rev. D 96, 083504 (2017).

[47] P. Friedrich and T. Prokopec, Kinetic theory and classical limit for real scalar quantum field in curved spacetime, Phys. Rev. D 98, 025010 (2018).

[48] S. R. de Groot, W. A. van Leeuwen, and Ch. G. van Weert, Relativistic Kinetic Theory: Principles and Applications (North-Holland Publishing Company, Amsterdam, 1980).

[49] C. Cercignani and G. M. Kremer, The Relativistic Boltzmann Equation: Theory and Applications (Birkhäuser Verlag, Basel, 2002).

[50] S. Pueblas and R. Scoccimarro, Generation of vorticity and velocity dispersion by orbit crossing, Phys. Rev. D 80, 043504 (2009).

[51] C. Uhlemann, Finding closure: Approximating VlasovPoisson using finitely generated cumulants, J. Cosmol. Astropart. Phys. 10 (2018) 030.

[52] A. Erschfeld and S. Floerchinger, Evolution of dark matter velocity dispersion, J. Cosmol. Astropart. Phys. 06 (2019) 039.

[53] P. McDonald, How to generate a significant effective temperature for cold dark matter, from first principles, J. Cosmol. Astropart. Phys. 04 (2011) 032.

[54] S. Floerchinger and E. Grossi, Causality of fluid dynamics for high-energy nuclear collisions, J. High Energy Phys. 08 (2018) 186.

[55] S. Floerchinger, N. Tetradis, and U. A. Wiedemann, Accelerating Cosmological Expansion from Shear and Bulk Viscosity, Phys. Rev. Lett. 114, 091301 (2015).

[56] D. Blas, S. Floerchinger, M. Garny, N. Tetradis, and U. A. Wiedemann, Large scale structure from viscous dark matter, J. Cosmol. Astropart. Phys. 11 (2015) 049.

[57] S. Floerchinger, M. Garny, N. Tetradis, and U.A. Wiedemann, Renormalization-group flow of the effective action of cosmological large-scale structures, J. Cosmol. Astropart. Phys. 01 (2017) 048.

[58] S. Floerchinger, M. Garny, A. Katsis, N. Tetradis, and U. A. Wiedemann, The dark matter bispectrum from effective viscosity and one-particle irreducible vertices, J. Cosmol. Astropart. Phys. 09 (2019) 047.

[59] P. L. Bhatnagar, E. P. Gross, and M. Krook, A model for collision processes in gases. I. Small amplitude processes in charged and neutral one-component systems, Phys. Rev. 94, 511 (1954).

[60] J. L. Anderson and H. R. Witting, A relativistic relaxation-time model for the Boltzmann equation, Physica (Amsterdam) 74, 466 (1974). 